\title{
Stronger Forms of Sensitivity for Measure-Preserving Maps and Semiflows on Probability Spaces
}

\author{
Risong $\mathrm{Li}^{1}$ and Yuming Shi ${ }^{2}$ \\ ${ }^{1}$ School of Science, Guangdong Ocean University, Zhanjiang 524025, China \\ ${ }^{2}$ Department of Mathematics, Shandong University, Jinan, Shandong 250100, China \\ Correspondence should be addressed to Risong Li; gdoulrs@163.com
}

Received 29 January 2014; Accepted 25 April 2014; Published 7 May 2014

Academic Editor: Miroslaw Lachowicz

Copyright (c) $2014 \mathrm{R}$. Li and Y. Shi. This is an open access article distributed under the Creative Commons Attribution License, which permits unrestricted use, distribution, and reproduction in any medium, provided the original work is properly cited.

\begin{abstract}
This paper is concerned with some stronger forms of sensitivity for measure-preserving maps and semiflows on probability spaces. A new form of sensitivity is introduced, called ergodic sensitivity. It is shown that, on a metric probability space with a fully supported measure, if a measure-preserving map is weak mixing, then it is ergodically sensitive and multisensitive; and if it is strong mixing, then it is cofinitely sensitive, where it is not required that the map is continuous and the space is compact. Similar results for measurepreserving semiflows are obtained, where it is required in a result about ergodic sensitivity that the space is compact in some sense and the semiflow is continuous. In addition, relationships between some sensitive properties of a map and its iterations are discussed, including syndetic sensitivity, cofinite sensitivity, ergodic sensitivity as well as usual sensitivity, $n$-sensitivity, and multisensitivity. Moreover, it is shown that multisensitivity, cofinite sensitivity, and ergodic sensitivity can be lifted up by a semiopen factor map.
\end{abstract}

\section{Introduction}

One of the most interesting characteristics of a dynamical system is when orbits of nearby points deviate after finite steps. This is also one of the important features of chaotic dynamical behaviors. It is termed as sensitive dependence on initial conditions (briefly, sensitivity). Sensitivity is a key notion when studying the complexity of a dynamical system. So, it is very important to study what systems have sensitive dependence. This problem has gained much attention recently (see [1-14]).

In [1], Abraham et al. proved that if a measure-preserving map $T$ on a metric probability space $(X, d, \mathscr{B}(X), \mu)$ with $\operatorname{supp} \mu=X$ is either topologically mixing or weak mixing and satisfies that for any nonempty open set $U \subset X$ and there exists a subsequence $\left\{n_{k}\right\}$ with positive upper density such that

$$
U \bigcap\left(\bigcap_{k \geq 0} T^{-n_{k}} U\right) \neq \emptyset,
$$

then $T$ is sensitive. In the same paper, they proved that if $T$ is strong mixing and $\sup \mu=X$, then it is sensitive; and if $T$ is an exact endomorphism and $\sup \mu=X$, then it is cofinitely sensitive. He et al. [8] showed that if a measurepreserving map $T$ (resp., a measure-preserving semiflow $\varphi$ ) on $(X, d, \mathscr{B}(X), \mu)$ with supp $\mu=X$ is weak mixing, then it is sensitive. In addition, if $X$ is a nontrivial metric space (i.e., a metric space is not reduced to a single point) and a map $f$ on $X$ is topologically mixing, then $f$ is sensitive [7, Proposition 7.2.14].

There are several ways to extend this notion. Here, we only list the following three ways:

(1) one may define $n$-sensitivity as it was done by Nemiskii and Stepanov in [15] and Ye and Zhang in [16];

(2) one may require that in any open subset $U$ there is a pair $(x ; y)$ which is a Li-Yorke pair as Akin and Kolyada in [17] did (see also recent work by Li et al. in [18], where a stronger form of sensitivity is defined);

(3) the third way is what we now consider in the present paper, that is, study $N_{f}(V, \delta)$.

Previously, the third way was considered by several scholars. More recently, Moothathu [12] studied continuous self-maps 
on compact metric spaces and initiated a preliminary study of stronger forms of sensitivity, including syndetic sensitivity, cofinite sensitivity, and multisensitivity. In particular, he showed that any syndetically transitive and nonminimal map is syndetically sensitive. This improves the result that if a continuous map is topologically transitive and has a dense set of periodic points in an infinite metric space, then it is sensitive [3]. Xiong [14] introduced the concept of $n$ sensitivity for continuous self-maps of a complete metric space. Later, Shao et al. [13] investigated some properties of $n$-sensitivity of continuous and surjective maps on a compact metric space. James et al. [10] introduced a notion, called measurable sensitivity and showed that a totally ergodic and measurably sensitive map is weakly mixing. More recently, Huang et al. [9] introduced the concepts of $\mu$-sensitivity, $n$-sensitivity for $\mu, \mu$-complexity, and $\mu$-equicontinuity for a measure-preserving and continuous map on a metric probability space $(X, d, \mathscr{B}(X), \mu)$ and presented a sufficient condition for $n$-sensitivity for $\mu$, where $X$ is a compact metric space. They proved that $\mu$-sensitivity is equivalent to pairwise sensitivity defined by Cadre and Jacob in [4].

In this paper, we introduce a new and stronger form of sensitivity, ergodic sensitivity, and present several sufficient conditions for multisensitivity, cofinite sensitivity, and ergodic sensitivity of measure-preserving maps and semiflows, where it is not required that maps and semiflows are continuous and spaces are compact. We show that, for a measure-preserving map on a metric probability space with a fully supported measure, if it is weak mixing, then it is ergodically sensitive and multisensitive; and if it is strong mixing, then it is cofinitely sensitive. Related problems for measure-preserving semiflow are also discussed. In addition, we consider the relationships between five forms of sensitivity (i.e., sensitivity, multisensitivity, cofinite sensitivity, syndetic sensitivity, ergodic sensitivity, and $n$-sensitivity) of a map $f$ and its iterations $f^{m}$ for $m \geq 2$.

The rest of this paper is organized as follows. In Section 2, we recall some basic concepts and lemmas and introduce a new and stronger form of sensitivity, called ergodic sensitivity. In Section 3, we give several sufficient conditions for multisensitivity, cofinite sensitivity, and ergodic sensitivity. Finally, we discuss the relationships between five forms of sensitivity of a map and its iterations in Section 4.

\section{Preliminaries}

In this section, we first introduce some notations and basic concepts, including a new and stronger form of sensitivity, called ergodic sensitivity, and then give two useful lemmas.

By $\mathbf{N}$ denote the set of all positive integers. Denote $\mathbf{Z}^{+}:=$ $\{0,1, \ldots\}, \mathbf{R}^{+}:=[0,+\infty)$, and $\mathbf{N}_{n}:=\{0,1, \ldots, n-1\}$. We will use $|A|$ to denote the cardinality of a set $A$.

We refer to $[12,19,20]$ for the following basic concepts. Let $(X, d)$ be a metric space, $\mathscr{B}(X)$ the sigma-algebra of Borel subsets of $X$, and $\mu$ a probability measure on $(X, \mathscr{B}(X))$. Then the space $X$ is called to be a metric probability space, denoted by the quadruple $(X, d, \mathscr{B}(X), \mu)$, which is often briefly denoted by the triple $(X, \mathscr{B}(X), \mu)$.
A measurable map $T$ is called measure-preserving on $(X, \mathscr{B}(X), \mu)$ if $\mu(B)=\mu\left(T^{-1}(B)\right)$ for any $B \in \mathscr{B}(X)$. A measurable semiflow $\varphi$ is called measure-preserving on $(X, \mathscr{B}(X), \mu)$ if $\mu(B)=\mu\left(\varphi_{t}^{-1}(B)\right)$ for any $B \in \mathscr{B}(X)$ and for any $t \in \mathbf{R}^{+}$.

The following concepts are about mixing properties of maps and semiflows in the measure-theoretical sense.

Definition 1. (i) A measure-preserving map $T$ on $(X, \mathscr{B}(X), \mu)$ is called weak mixing and strong mixing if, for any $A, B \in \mathscr{B}(X)$, the following two equalities hold, respectively:

$$
\begin{gathered}
\lim _{n \rightarrow \infty} \frac{1}{n} \sum_{i=0}^{n-1}\left|\mu\left(A \bigcap T^{-i}(B)\right)-\mu(A) \mu(B)\right|=0, \\
\lim _{n \rightarrow \infty} \mu\left(A \bigcap T^{-n}(B)\right)=\mu(A) \mu(B) .
\end{gathered}
$$

(ii) A measure-preserving semiflow $\varphi$ on $(X, \mathscr{B}(X), \mu)$ is called weak mixing and strong mixing if, for any $A, B \in$ $\mathscr{B}(X)$, the following two equalities hold, respectively:

$$
\begin{gathered}
\lim _{t \rightarrow \infty} \frac{1}{t} \int_{0}^{t}\left|\mu\left(A \bigcap \varphi_{s}^{-1}(B)\right)-\mu(A) \mu(B)\right| d s=0, \\
\lim _{t \rightarrow \infty} \mu\left(A \bigcap \varphi_{t}^{-1}(B)\right)=\mu(A) \mu(B) .
\end{gathered}
$$

The following concepts describe three different forms of transitivity of a map $f: X \rightarrow X$ and a semiflow $\varphi: \mathbf{R}^{+} \times X \rightarrow$ $X$ in the topological sense. For convenience, denote

$$
\begin{gathered}
N_{f}(U, V):=\left\{n \in \mathbf{N}: T^{n}(U) \cap V \neq \emptyset\right\}, \\
N_{\varphi}(U, V):=\left\{t>0: \varphi_{t}(U) \cap V \neq \emptyset\right\}
\end{gathered}
$$

for any sets $U, V \subset X$.

Definition 2. Let $f: X \rightarrow X$ be a map and $X$ a metric space.

(i) The map $f$ is said to be topologically transitive and topologically mixing on $X$ if, for any pair of nonempty open sets $U, V \subset X$, the following conditions hold, respectively: $N_{f}(U, V) \neq \emptyset$ and $N_{f}(U, V) \supset\{k, k+$ $1, \ldots\}$ for some integer $k \geq 1$.

(ii) The map $f$ is said to be topologically weakly mixing on $X$ if $f \times f$ is topologically transitive on the product space $X \times X$.

Clearly, topological mixing is stronger than topologically weak mixing, and topologically weak mixing is stronger than topological transitivity. There are other two different forms of transitivity: syndetic transitivity [15] and topological ergodicity [21], which are not considered in the present paper.

Their corresponding concepts to semiflows are given as follows.

Definition 3. Let $\varphi: \mathbf{R}^{+} \times X \rightarrow X$ be a semiflow and $X$ a metric space. 
(i) The semiflow $\varphi$ is said to be topologically transitive and topologically mixing on $X$ if, for any pair of nonempty open sets $U, V \subset X$, the following conditions hold, respectively: $N_{\varphi}(U, V) \neq \emptyset$ and $N_{\varphi}(U, V)$ ว $[L,+\infty)$ for some constant $L>0$.

(ii) A semiflow $\varphi$ is said to be topologically weakly mixing on $X$ if $\varphi \times \varphi$ is topologically transitive on the product space $X \times X$.

Let $S$ be a subset of $\mathbf{Z}^{+}$(resp., a Lebesgue measurable subset of $\mathbf{R}^{+}$). Its upper and lower densities are defined, respectively, by

$$
\begin{aligned}
& \bar{d}(S):=\limsup _{k \rightarrow \infty} \frac{1}{k}\left|S \bigcap N_{k}\right|, \\
& \underline{d}(S):=\liminf _{k \rightarrow \infty} \frac{1}{k}\left|S \bigcap N_{k}\right|
\end{aligned}
$$

(resp., $\lim \sup _{t \rightarrow \infty}(1 / t) l(S \cap[0, t])$ and $\lim \inf _{t \rightarrow \infty}(1 / t) l(S \cap$ $[0, t])$, where $l(S)$ is the Lebesgue measure of $S[8])$, and its density is defined by

$$
d(S):=\lim _{k \rightarrow \infty} \frac{1}{k}\left|S \bigcap N_{k}\right|
$$

(resp., $\left.\lim _{t \rightarrow \infty}(1 / t) l(S \cap[0, t])\right)$ and if it exists.

According to the classical definition, a map $f$ (resp., a semiflow $\varphi$ ) is sensitive in $X$ if there is a constant $\delta>0$ such that, for any $x \in X$ and any open neighborhood $V_{x}$ of $x$, there is $n \in \mathbf{Z}^{+}$(resp., $t \in \mathbf{R}^{+}$) such that $\sup \left\{d\left(f^{n}(x), f^{n}(y)\right): y \in\right.$ $\left.V_{x}\right\}>\delta$ (resp., $\sup \left\{d\left(\varphi_{t}(x), \varphi_{t}(y)\right): y \in V_{x}\right\}>\delta$ ), where $\delta$ is called a constant of sensitivity. Now, we write this in a slightly different way. For $V \subset X$ and $\delta>0$, denote

$$
\begin{array}{r}
N_{f}(V, \delta):=\left\{n \in \mathbf{Z}^{+}: \text {there exist } x, y \in V\right. \\
\text { with } \left.d\left(f^{n}(x), f^{n}(y)\right)>\delta\right\}, \\
N_{\varphi}(V, \delta):=\left\{t \in \mathbf{R}^{+}: \text {there exist } x, y \in V\right. \\
\text { with } \left.d\left(\varphi_{t}(x), \varphi_{t}(y)\right)>\delta\right\} .
\end{array}
$$

In terms of these notations, the above sensitivity properties can be equivalently defined as [12]

(1) $f$ (resp., $\varphi$ ) is sensitive in $X$ if there is a constant $\delta>0$ such that $N_{f}(V, \delta)$ (resp., $N_{\varphi}(V, \delta)$ ) is nonempty for any nonempty open set $V \subset X$.

In [12], Moothathu gave the following three stronger forms of sensitivity:

(2) $f$ (resp., $\varphi$ ) is cofinitely sensitive in $X$ if there is a constant $\delta>0$ such that $N_{f}(V, \delta) \supset[n,+\infty) \bigcap \mathbf{N}$ for some $n \geq 1$ (resp., $N_{\varphi}(V, \delta) \supset[t,+\infty)$ for some $t>0$ ) for any nonempty open subset $V \subset X$;

(3) $f$ (resp., $\varphi$ ) is multisensitive in $X$ if there is a constant $\delta>0$ such that $\bigcap_{i=1}^{k} N_{f}\left(V_{i}, \delta\right) \neq \emptyset$ (resp., $\left.\bigcap_{i=1}^{k} N_{\varphi}\left(V_{i}, \delta\right) \neq \emptyset\right)$ for each $k \geq 1$ and any nonempty open sets $V_{1}, V_{2}, \ldots, V_{k} \subset X$;
(4) $f$ is syndetically sensitive in $X$ if there is a constant $\delta>0$ such that $N_{f}(V, \delta)$ is a syndetic set for any nonempty open set $V \subset X$.

Motivated by the idea in the definition of topological ergodicity introduced by Akin [21], we now introduce another stronger form of sensitivity as follows.

(5) $f$ (resp., $\varphi$ ) is called to be ergodically sensitive in $X$ if there is a constant $\delta>0$ such that $N_{f}(V, \delta)$ (resp., $\left.N_{\varphi}(V, \delta)\right)$ has a positive upper density for any nonempty open subset $V \subset X$.

For convenience, such a constant $\delta$ in the above definitions is called a sensitive constant of $f$ with respect to the corresponding sensitive forms.

Remark 4. In [12], it is required that the map is continuous and the space is compact in the definitions of the concepts in (9)-(25) as well as in the definitions of the three concepts given in Definition 2.

By the above definitions, it can be easily implied that

cofinitely sensitive $\Longrightarrow$ syndetically sensitive

$$
\Longrightarrow \text { ergodically sensitive } \Longrightarrow \text { sensitive. }
$$

So, cofinite sensitivity is the strongest one among the above five different forms of sensitivity.

Definition 5. Let $(X, d)$ be a nontrivial metrics space and $f$ : $X \rightarrow X$ a map. For a given integer $n \geq 2$, the system $(X, f)$ or the map $f$ is said to be $n$-sensitive if there exists a constant $\delta>0$ such that, for any nonempty and open set $U$, there are distinct points $x_{1}, x_{2}, \ldots, x_{n} \in U$ and some $m \in Z^{+}$satisfying that $d\left(f^{m}\left(x_{i}\right), f^{m}\left(x_{j}\right)\right) \geq \delta$ for $1 \leq i<j \leq n$. Such a constant $\delta$ is called an $n$-sensitive constant of $f$.

It is clear that 2 -sensitivity is just sensitivity. For any given $n \geq 2$, there exists a minimal system, that is, $n$-sensitive, but not $(n+1)$-sensitive (see [13]).

Remark 6. In the case that $X$ is a locally connected and compact nontrivial metric space, Shao et al. [13] showed that if a continuous and surjective map $f: X \rightarrow X$ is sensitive, then it is $n$-sensitive for all $n \geq 2$. Note that if the assumptions that $X$ is compact and $f$ is surjective are removed, then the result still holds. This can be easily verified by the method used in the proof of Proposition 4.1 in [14]. Consequently, if $f$ is multisensitive, then it is $n$-sensitive for each $n \geq 2$ in this case.

To end this section, we introduce two useful lemmas. A set $S \subset \mathbf{Z}^{+}$(resp., $S \subset \mathbf{R}^{+}$) is called relatively dense in $\mathbf{Z}^{+}$ (resp., $\mathbf{R}^{+}$) if there is $n \in \mathbf{N}$ (resp., $L>0$ ) such that, for any $k \in \mathbf{Z}^{+}$(resp., $t \in \mathbf{R}^{+}$), we have $S \cap\{k, k+1, \ldots, k+n-1\} \neq \emptyset$ (resp., $S \cap(t, t+L) \neq \emptyset$ ). Clearly, the relative density of a set in $\mathrm{Z}^{+}$is equivalent to its syndedicity. 
Lemma 7 (see [19]). Let $T$ be a measure-preserving map on $(X, \mathscr{B}(X), \mu)$. For any $A \in \mathscr{B}(X)$, if $\mu(A)>0$, then, for any $\varepsilon>0$, the set $S=\left\{k \in \mathbf{N}: \mu\left(A \bigcap T^{-k}(A)\right) \geq(\mu(A))^{2}-\varepsilon\right\}$ is relatively dense in $\mathbf{N}$.

Lemma 8 (see [15]). Let $\varphi$ be a measure-preserving semiflow on $(X, \mathscr{B}(X), \mu)$. For any $A \in \mathscr{B}(X)$, if $\mu(A)>0$, then the set $S=\left\{t \in \mathbf{R}^{+}: \mu\left(A \bigcap \varphi_{t}^{-1}(A)\right)>\lambda(\mu(A))^{2}\right\}(\lambda<1)$ is relatively dense in $\mathbf{R}^{+}$.

\section{Sufficient Conditions for Multisensitivity, Cofinite Sensitivity, and Ergodic Sensitivity}

In this section, we will give several sufficient conditions for multisensitivity, cofinite sensitivity, and ergodic sensitivity of measure-preserving maps and semiflows. This section is divided into three subsections.

3.1. Multisensitivity. In this subsection, we first show that multisensitivity can be lifted up by a semiopen factor map and then give a sufficient condition for multisensitivity of measure-preserving maps (resp., semiflows).

In [13], the authors proved that $n$-sensitivity can be lifted up by a semiopen factor map, where a map is called semiopen if the image of any nonempty open set contains a nonempty open subset. Now, we show that multisensitivity has the same property.

Let $f: X \rightarrow X$ and $g: Y \rightarrow Y$ be maps, where $X$ and $Y$ are metric spaces. If there exists a continuous and surjective map $\pi: X \rightarrow Y$ such that $\pi \circ f=g \circ \pi$, then $(Y, g)$ is said to be a factor of the system $(X, f)$, and $(X, f)$ is said to be an extension of $(Y, g)$, while $\pi$ is said to be a factor map between $(X, f)$ and $(Y, g)$.

Proposition 9. Let $(X, d)$ and $(Y, e)$ be nontrivial metric spaces, let $f: X \rightarrow X$ and $g: Y \rightarrow Y$ be maps, and let $\pi: X \rightarrow Y$ be a semiopen factor map between $(X, f)$ and $(Y, g)$. If $g$ is multisensitive, then so is $f$.

Proof. Suppose that $g$ is multisensitive with sensitive constant $\delta>0$. By the continuity of $\pi$, there exists a constant $\delta^{\prime}>0$ such that if $e\left(y_{1}, y_{2}\right)>\delta$ for $y_{1}, y_{2} \in Y$, then $d\left(x_{1}, x_{2}\right)>\delta^{\prime}$, where $\pi\left(x_{i}\right)=y_{i}, i=1,2$. Let $n \geq 1$ be any given integer and $U_{i}$ any nonempty open set in $X$ for each $1 \leq i \leq n$. Since $\pi$ is semiopen, $\pi\left(U_{i}\right)$ contains a nonempty open subset $V_{i}$ for each $1 \leq i \leq n$. Therefore, $\bigcap_{i=1}^{n} N_{g}\left(V_{i}, \delta\right) \neq \emptyset$ implies $\bigcap_{i=1}^{n} N_{f}\left(U_{i}, \delta^{\prime}\right) \neq \emptyset$. Thus, the proof is complete.

Next, we study sufficient conditions for multisensitivity.

Lemma 10. Let $(X, d)$ be a nontrivial metric space. If a map $f$ : $X \rightarrow X$ satisfies that $\underbrace{f \times f \times \cdots \times f}_{k}$ is topologically transitive for each integer $k \geq 1$, then $f$ is multisensitive in $X$.

Proof. As $X$ is not reduced to a single point, there exists a constant $\delta>0$ such that, for every $x \in X$, there is $y \in X$ satisfying $d(x, y)>3 \delta$. We will remark that this claim will be repeatedly used in this section.
Fix any integer $k \geq 1$ and let $V_{i} \subset X, 1 \leq i \leq k$, be any bounded and nonempty open sets with $\operatorname{diam}\left(V_{i}\right)<\delta$, where $\operatorname{diam}\left(V_{i}\right):=\sup _{x, y \in V_{i}}\{d(x, y)\}$ is the diameter of $V_{i}$. Then there exists a nonempty open subset $U_{i}$ such that

$$
d\left(U_{i}, V_{i}\right)>\delta, \quad 1 \leq i \leq k .
$$

Since $\underbrace{f \times f \times \cdots \times f}_{2 k}$ is topologically transitive, one has

$$
\begin{gathered}
\underbrace{(f \times f \times \cdots \times f)^{n}}_{2 k}\left(\left(V_{1} \times V_{1}\right) \times\left(V_{2} \times V_{2}\right) \times \cdots \times\left(V_{k} \times V_{k}\right)\right) \\
\bigcap\left(\left(V_{1} \times U_{1}\right) \times\left(V_{2} \times U_{2}\right) \times \cdots \times\left(V_{k} \times U_{k}\right)\right) \neq \emptyset
\end{gathered}
$$

for some integer $n \geq 1$. This implies that $f^{n}\left(V_{i}\right) \cap V_{i} \neq \emptyset$ and $f^{n}\left(V_{i}\right) \cap U_{i} \neq \emptyset$, and consequently there exist $x_{i}, x_{i}^{\prime} \in V_{i}$ such that $f^{n}\left(x_{i}\right) \in V_{i}$ and $f^{n}\left(x_{i}^{\prime}\right) \in U_{i}$ for $1 \leq i \leq k$. So, it follows from (9) that $d\left(f^{n}\left(x_{i}\right), f^{n}\left(x_{i}^{\prime}\right)\right)>\delta$ for $1 \leq i \leq k$. This yields

$$
\bigcap_{i=1}^{k} N_{f}\left(V_{i}, \delta\right) \neq \emptyset .
$$

Therefore, $f$ is multisensitive in $X$. The proof is complete.

Remark 11. It is known that a continuous map on a compact space is topologically weak mixing if and only if $\underbrace{f \times f \times \cdots \times f}_{k}$ is topologically transitive for each $k \geq 2$.

(1) In [22, Theorem 3.1], it was shown that if $f$ is continuous and topologically mixing on a compact metric space $X$, then it is sensitive. Since the topological mixing is stronger than the topological weak mixing, Lemma 10 relaxes the conditions of [22, Theorem 3.1] and improves it by noting that it is not required that the space is compact and the map is continuous.

(2) In [12], Moothathu claimed that if a continuous map $f$ is topologically weak mixing on a compact metric space, then it is multisensitive in $X$. So, Lemma 10 relaxes the conditions of this result.

Theorem 12. Let $(X, d)$ be a nontrivial metric space and let $T$ be a measure-preserving map on $(X, d, \mathscr{B}(X), \mu)$. If $T$ is weak mixing and supp $\mu=X$, then $T^{n}$ is multisensitive in $X$ for every integer $n \geq 1$.

Proof. By the definition, one can easily prove that $T$ is weak mixing if and only if $T^{n}$ is too for each $n \geq 1$. So it suffices to show that $T$ is multisensitive in $X$. Since $T$ is weak mixing, $\underbrace{T \times T \times \cdots T}_{2 k}$ is weak mixing for each $k \geq 1$ by Theorem 1.24 in [15]. Further, every nonempty open set in $X$ has a positive measure because of $\operatorname{supp} \mu=X$. It follows that $\underbrace{T \times T \times \cdots T}_{2 k}$ is topologically transitive for each $k \geq 1$. Therefore, $T$ is multisensitive in $X$ by Lemma 10. This completes the proof. 
In order to extend the above result for measure-preserving maps to measure-preserving semiflows, we first show the following five lemmas.

Lemma 13. Let $(X, d)$ be a nontrivial metric space. If a semiflow $\varphi: \mathbf{R}^{+} \times X \rightarrow X$ satisfies that $\underbrace{\varphi \times \varphi \times \cdots \times \varphi}$ is topologically transitive for each integer $k \geq 1$, then it is multisensitive in $X$.

Proof. With a similar argument to that used in the proof of Lemma 10, one can easily prove Lemma 13. So its details are omitted.

Lemma 14. Let $\varphi$ be a measure-preserving semiflow on $(X, d, \mathscr{B}(X), \mu)$ and $\Omega$ a semialgebra that generates $\mathscr{B}(X)$. Then $\varphi$ is weak mixing if and only if, for any $A, B \in \Omega$, we have

$$
\lim _{t \rightarrow \infty} \frac{1}{t} \int_{0}^{t}\left|\mu\left(A \bigcap \varphi_{s}^{-1}(B)\right)-\mu(A) \mu(B)\right| d s=0
$$

Proof. The proof is similar to that of Theorem 1.17 in [20] and then is omitted.

Lemma 15. The following are equivalent:

(i) $\lim _{t \rightarrow \infty}(1 / t) \int_{0}^{t}|f(s)| d s=0$;

(ii) there exists a subset $S \subset \mathbf{R}^{+}$of density zero such that $\lim _{t \rightarrow \infty} f(t)=0$ provided $t \notin S$;

(iii) $\lim _{t \rightarrow \infty}(1 / t) \int_{0}^{t}|f(s)|^{2} d s=0$.

Proof. The proof is similar to that of Theorem 1.20 in [20] and then is omitted here.

The following lemma can be directly derived from Lemma 15 with $f(s)=\mu\left(\varphi_{s}^{-1}(A) \bigcap B\right)-\mu(A) \mu(B)$.

Lemma 16. Let $\varphi$ be a measure-preserving semiflow on $(X, d, \mathscr{B}(X), \mu)$. Then the following are equivalent:

(i) $\varphi$ is weak mixing;

(ii) for every pair of sets $A, B \in \mathscr{B}(X)$, there is a subset $S(A, B) \subset \mathbf{R}^{+}$of density zero such that

$$
\lim _{\mathbf{R}^{+} \backslash S(A, B) \ni t \rightarrow \infty} \mu\left(\varphi_{t}^{-1}(A) \bigcap B\right)=\mu(A) \mu(B) ;
$$

(iii) for every pair of sets $A, B \in \mathscr{B}(X)$, one has

$$
\lim _{t \rightarrow \infty} \frac{1}{t} \int_{0}^{t}\left|\mu\left(\varphi_{s}^{-1}(A) \bigcap B\right)-\mu(A) \mu(B)\right|^{2} d s=0 .
$$

Lemma 17. Let $\varphi$ be a measure-preserving semiflow on $(X, d, \mathscr{B}(X), \mu)$. Then $\varphi$ is weak mixing if and only if so is $\varphi \times \varphi$.
Proof. First consider the necessity. Suppose that $\varphi$ is weak mixing. Fix any sets $A, B, C, D \in \mathscr{B}(X)$. Then by Lemma 16 there exist subsets $S_{1}, S_{2} \subset \mathbf{R}^{+}$of density zero such that

$$
\begin{array}{r}
\lim _{\mathbf{R}^{+} \backslash S_{1} \ni t \rightarrow \infty} \mu\left(\varphi_{t}^{-1}(A) \bigcap B\right)=\mu(A) \mu(B), \\
\lim _{\mathbf{R}^{+} \backslash S_{2} \ni t \rightarrow \infty} \mu\left(\varphi_{t}^{-1}(C) \bigcap D\right)=\mu(C) \mu(D),
\end{array}
$$

which yield that

$$
\begin{aligned}
& \lim _{\mathbf{R}^{+} \backslash\left(S_{1} \cup S_{2}\right) \ni t \rightarrow \infty}(\mu \times \mu)\left((\varphi \times \varphi)_{t}^{-1}(A \times C) \bigcap(B \times D)\right) \\
& =\lim _{\mathbf{R}^{+} \backslash\left(S_{1} \cup S_{2}\right) \ni t \rightarrow \infty} \mu\left(\varphi_{t}^{-1}(A) \bigcap B\right) \mu\left(\varphi_{t}^{-1}(C) \bigcap D\right) \\
& =\mu(A) \mu(B) \mu(C) \mu(D) \\
& =(\mu \times \mu)(A \times C)(\mu \times \mu)(B \times D) .
\end{aligned}
$$

So, by Lemma 15 one has

$$
\begin{aligned}
\lim _{t \rightarrow \infty} \frac{1}{t} \int_{0}^{t} \mid & (\mu \times \mu)(\varphi \times \varphi)_{s}^{-1}((A \times C) \bigcap(B \times D)) \\
& -(\mu \times \mu)(A \times C)(\mu \times \mu)(B \times D) \mid d s=0 .
\end{aligned}
$$

Since the measurable rectangles form a semialgebra that generates $\mathscr{B}(X) \times \mathscr{B}(X)$. Therefore, $\varphi \times \varphi$ is weak mixing by Lemma 14.

Next, we consider the sufficiency. Suppose that $\varphi \times \varphi$ is weak mixing. Fix any sets $A, B \in \mathscr{B}(X)$. It is evident that

$$
\begin{gathered}
\mu\left(\varphi_{s}^{-1}(A) \bigcap B\right)=(\mu \times \mu)\left((\varphi \times \varphi)_{s}^{-1}(A \times X) \bigcap(B \times X)\right) \\
(\mu \times \mu)(A \times X)(\mu \times \mu)(B \times X)=\mu(A) \mu(B),
\end{gathered}
$$

which, together with the assumption that $\varphi \times \varphi$ is weak mixing, imply that

$$
\begin{aligned}
& \lim _{t \rightarrow \infty} \frac{1}{t} \int_{0}^{t}\left|\mu\left(\varphi_{s}^{-1}(A) \bigcap B\right)-\mu(A) \mu(B)\right| d s \\
&=\lim _{t \rightarrow \infty} \frac{1}{t} \int_{0}^{t} \mid(\mu \times \mu)\left((\varphi \times \varphi)_{s}^{-1}(A \times X) \bigcap(B \times X)\right) \\
& \quad-(\mu \times \mu)(A \times X)(\mu \times \mu)(B \times X) \mid d s=0 .
\end{aligned}
$$

Hence, $\varphi$ is weak mixing. The entire proof is complete.

Theorem 18. Let $(X, d)$ be a nontrivial metric space and $\varphi$ a measure-preserving semiflow on $(X, d, \mathscr{B}(X), \mu)$. If $\varphi$ is weak mixing and supp $\mu=X$, then $\varphi$ is multisensitive in $X$.

Proof. With a similar argument to that used in the proof of Theorem 12, one can easily show this theorem by Lemmas 13 and 17. This completes the proof. 
3.2. Cofinite Sensitivity. In this subsection, we first show that cofinite sensitivity can be lifted by a semiopen factor map and then give a sufficient condition for cofinite sensitivity of measure-preserving maps (resp., semiflows).

Proposition 19. Let $X$ and $Y$ be nontrivial metric spaces, let $f: X \rightarrow X$ and $g: Y \rightarrow Y$ be maps, and let $\pi: X \rightarrow Y$ be a semiopen factor map between $(X, f)$ and $(Y, g)$. If $g$ is cofinitely sensitive, then so is $f$.

Proof. The proof is similar to that of Proposition 9 and so its details are omitted.

Lemma 20. Let $(X, d)$ be a nontrivial metric space. If a map $f: X \rightarrow X$ is topologically mixing, then it is cofinitely sensitive in $X$.

Proof. As is shown in Lemma 10, there exists a constant $\delta>$ 0 such that, for every nonempty open set $V \subset X$ with $\operatorname{diam}(V)<\delta$, there exists a nonempty open set $U$ satisfying

$$
d(U, V)>\delta \text {. }
$$

Since $f: X \rightarrow X$ is topologically mixing, there is an integer $n_{0} \geq 1$ such that

$$
f^{n}(V) \bigcap V \neq \emptyset, \quad f^{n}(V) \bigcap U \neq \emptyset, \quad \forall n \geq n_{0},
$$

which, together with (20), yield that $N_{f}(V, \delta)$ ว $\left[n_{0},+\infty\right) \bigcap \mathbf{N}$. Therefore, $f$ is cofinitely sensitive in $X$. This completes the proof.

Remark 21. Proposition 2 in [12] shows that if a map $f$ is topologically mixing and continuous in a compact metric space $X$, then $f$ is cofinitely sensitive in $X$. Note that the compactness of the space $X$ and the continuity of the map $f$ are not required in Lemma 20. So Lemma 20 relaxes the conditions of this proposition.

This result can be extended to semiflows.

Lemma 22. Let $(X, d)$ be a nontrivial metric space. If a semiflow $\varphi: \mathbf{R}^{+} \times X \rightarrow X$ is topologically mixing, then it is cofinitely sensitive in $X$.

Proof. The proof is similar to that of Lemma 20 and so it is omitted.

Theorem 23. Let $(X, d)$ be a nontrivial metric space and let $T$ be a measure-preserving map on $(X, d, \mathscr{B}(X), \mu)$. If $T$ is strong mixing and supp $\mu=X$, then $T^{n}$ is cofinitely sensitive in $X$ for each integer $n \geq 1$.

The above theorem follows from Proposition 2.2 in [10]. For completeness, we now give a different proof here.

Proof. By the definition of strong mixing, it can be easily seen that $T$ is strong mixing if and only if $T^{n}$ is too for each $n \geq 1$. So it suffices to show that $T$ is cofinitely sensitive in $X$. Because of supp $\mu=X$, every nonempty open set in $X$ has a positive measure. So $T$ is topologically mixing. Consequently, by Lemma 20, $T$ is cofinitely sensitive in $X$. Thus, the proof is complete.

Theorem 24. Let $(X, d)$ be a nontrivial metric space and let $\varphi$ be a measure-preserving semiflow on $(X, d, \mathscr{B}(X), \mu)$. If $\varphi$ is strong mixing and supp $\mu=X$, then $\varphi$ is cofinitely sensitive in $X$.

Proof. With a similar argument to that used in the proof of Theorem 23 and by Lemma 22, one can easily show that this theorem holds. The proof is complete.

3.3. Ergodic Sensitivity. In the final subsection, we will first show that ergodic sensitivity can be lifted by a semiopen factor map and then consider ergodic sensitivity for measurepreserving maps and semiflows on a probability space and give a sufficient condition for each of them.

Proposition 25. Let $X$ and $Y$ be nontrivial metric spaces, let $f: X \rightarrow X$ and $g: Y \rightarrow Y$ be maps, and let $\pi: X \rightarrow Y$ be a semiopen factor map between $(X, f)$ and $(Y, g)$. If $g$ is ergodically sensitive, then so is $f$.

Proof. The proof is similar to that of Proposition 9 and so its details are omitted.

Lemma 26. Let $(X, d)$ be a nontrivial metric space and $T$ a measure-preserving map on $(X, d, \mathscr{B}(X), \mu)$. If $T$ is not ergodically sensitive in $X$ and supp $\mu=X$, then there exist $a$ constant $\delta>0$ and two disjoint and nonempty open sets $U$ and $V$ in $X$ such that

$$
\bar{d}\left(S \bigcap\left(\mathbf{Z}^{+} \backslash N_{T}(W, \delta)\right)\right)>0
$$

for some nonempty open set $W \subset X$, where

$$
S=\left\{i \in \mathbf{Z}^{+}: T^{i}(V) \bigcap U=\emptyset\right\} .
$$

Proof. As is shown in the proof of Lemma 10, there exists a constant $\delta>0$ such that, for every $x \in X$, there is $y \in X$ with $d(x, y)>3 \delta$. Since $T$ is not ergodically sensitive in $X$, there exists a nonempty open set $W \subset X$ such that $\bar{d}\left(N_{T}(W, \delta)\right)=0$ and so

$$
d\left(N_{T}(W, \delta)\right)=0
$$

It is clear that

$$
\operatorname{diam}\left(T^{n}(W)\right) \leq \delta, \quad \forall n \in \mathbf{Z}^{+} \backslash N_{T}(W, \delta)
$$

Fix a point $x \in W$ and take a constant $0<\varepsilon<\delta$ with the open ball $B(x, \varepsilon) \subset W$. Then $\mu(B(x, \varepsilon))>0$ because of supp $\mu=X$. By Lemma 7, the set

$$
S_{1}=\left\{i \in \mathbf{Z}^{+}: B(x, \varepsilon) \bigcap T^{-i}(B(x, \varepsilon)) \neq \emptyset\right\}
$$

is relatively dense in $\mathbf{Z}^{+}$. Now, for any $i \in S_{1}$, take

$$
z_{i} \in B(x, \varepsilon) \bigcap T^{-i}(B(x, \varepsilon))
$$


which implies that

$$
T^{i}\left(z_{i}\right) \in B(x, \varepsilon) \bigcap T^{i}(B(x, \varepsilon)) .
$$

So it follows from (25) that, for any $y \in B(x, \varepsilon)$ and any $k \in$ $S_{1} \cap\left(\mathbf{Z}^{+} \backslash N_{T}(W, \delta)\right)$,

$$
\begin{aligned}
d\left(T^{k}(y), x\right) & \leq d\left(T^{k}(y), T^{k}\left(z_{k}\right)\right)+d\left(T^{k}\left(z_{k}\right), x\right) \\
& \leq \delta+\varepsilon<2 \delta .
\end{aligned}
$$

This means that $T^{k}(B(x, \varepsilon)) \subset B(x, 2 \delta)$ for any $k \in S_{1} \bigcap\left(\mathbf{Z}^{+} \backslash\right.$ $N_{T}(W, \delta)$ ).

Set $V=B(x, \varepsilon)$ and $U=X \backslash \bar{B}(x, 2 \delta)$. Then $U$ and $V$ are disjoint and nonempty open sets, and $T^{k}(V) \bigcap U=\emptyset$ for any $k \in S_{1} \cap\left(\mathbf{Z}^{+} \backslash N_{T}(W, \delta)\right)$, which implies that

$$
S_{1} \bigcap\left(\mathbf{Z}^{+} \backslash N_{T}(W, \delta)\right) \subset S,
$$

and consequently

$$
s \bigcap\left(\mathbf{Z}^{+} \backslash N_{T}(W, \delta)\right)>S_{1} \bigcap\left(\mathbf{Z}^{+} \backslash N_{T}(W, \delta)\right) .
$$

As the lower density of $S_{1}$ is positive which implies that the upper density of

$$
S \cap\left(Z^{+} \backslash N_{T}(W, \delta)\right)
$$

is positive, since the upper density of

$$
Z^{+} \backslash N_{T}(W, \delta)
$$

is 1 , the proof is complete.

By the Birkhoff ergodic theorem and Lemma 26 one can prove the following theorem. For completeness, we give another proof of Theorem 27.

Theorem 27. Let $(X, d)$ be a nontrivial metric space and let $T$ be a measure-preserving map on $(X, d, \mathscr{B}(X), \mu)$. If T is weak mixing and supp $\mu=X$, then $T^{n}$ is ergodically sensitive in $X$ for each integer $n \geq 1$.

Proof. As is shown in the proof of Theorem 12, $T$ is weak mixing if and only if $T^{n}$ is too for each $n \geq 1$. So it suffices to show that $T$ is ergodically sensitive in $X$.

Suppose on the contrary that $T$ is not ergodically sensitive in $X$. Then, by Lemma 26 there exist a constant $\delta>0$ and two disjoint and nonempty open sets $U$ and $V$ in $X$ such that the set $S \cap\left(\mathbf{Z}^{+} \backslash N_{T}(W, \delta)\right)$ has a positive upper density for some nonempty open set $W \subset X$, where $S=\left\{i \in \mathbf{Z}^{+}: T^{i}(V) \cap U=\right.$ $\emptyset\}$. Then

$$
V \bigcap T^{-k}(U)=\emptyset, \quad \forall k \in S,
$$

which implies that $\mu\left(V \cap T^{-k}(U)\right)=0$ for each $k \in S$. Thus, one has that

$$
\begin{aligned}
\sum_{i=0}^{n-1} \mid \mu & \left(V \bigcap T^{-i}(U)\right)-\mu(V) \mu(U) \mid \\
& \geq\left|S \bigcap N_{n}\right| \mu(V) \mu(U) \\
& \geq\left|S \bigcap\left(\mathbf{Z}^{+} \backslash N_{T}(W, \delta)\right) \bigcap N_{n}\right| \mu(V) \mu(U) .
\end{aligned}
$$

Further, $\mu(V) \mu(U)>0$ since supp $\mu=X$, and $U$ and $V$ are nonempty open sets. Consequently,

$$
\begin{aligned}
& \limsup _{n \rightarrow \infty} \frac{1}{n} \sum_{k=0}^{n-1}\left|\mu\left(V \bigcap T^{-k}(U)\right)-\mu(V) \mu(U)\right| \\
& \geq \mu(V) \mu(U) \bar{d}\left(S \bigcap\left(\mathbf{Z}^{+} \backslash N_{T}(W, \delta)\right)\right)>0 .
\end{aligned}
$$

This is a contradiction since $T$ is weak mixing. Therefore, $T$ is ergodically sensitive in $X$. This completes the proof.

Remark 28. Syndetic sensitivity implies ergodic sensitivity. However, Moothathu gave an example of a sensitive map that is not ergodically sensitive in Theorem 7 in [12].

Lemma 29. Let $(X, d)$ be a nontrivial metric space, whose bounded and closed subsets are compact, and let $\varphi$ be a continuous measure-preserving semiflow on $(X, d, \mathscr{B}(X), \mu)$. If $\varphi$ is not ergodically sensitive in $X$ and $\operatorname{supp} \mu=X$, then there exist two disjoint and nonempty open sets $U, V$ in $X$ such that $\bar{d}(S)>0$, where

$$
S=\left\{t \in \mathbf{R}^{+}: V \bigcap \varphi_{t}^{-1}(U)=\emptyset\right\} .
$$

Proof. As is shown in the proof of Lemma 10, there exists a constant $\delta>0$ such that, for every $x \in X$, there is $y \in X$ with $d(x, y)>3 \delta$. By the assumption that $\varphi$ is not ergodically sensitive in $X$, there exists a nonempty open set $W \subset X$ such that $\bar{d}\left(N_{\varphi}(W, \delta)\right)=0$, and so $d\left(N_{\varphi}(W, \delta)\right)=0$. Consequently, $d\left(\mathbf{R}^{+} \backslash N_{\varphi}(W, \delta)\right)=1$.

Fix any $x \in W$. There exists a positive constant $\varepsilon<\delta$ such that $B(x, \varepsilon) \subset W$. As supp $\mu=X$, we have $\mu(B(x, \varepsilon))>0$. Take $V=B(x, \varepsilon)$. By Lemma 8 , the set

$$
\begin{aligned}
S_{1} & =\left\{t \in \mathbf{R}^{+}: V \bigcap \varphi_{t}(V) \neq \emptyset\right\} \\
& =\left\{t \in \mathbf{R}^{+}: V \bigcap \varphi_{t}^{-1}(V) \neq \emptyset\right\}
\end{aligned}
$$

is relatively dense in $\mathbf{R}^{+}$; that is, there exists $L>0$ such that

$$
S_{1} \bigcap(t, t+L) \neq \emptyset, \quad \forall t \in \mathbf{R}^{+} .
$$

For any $t \in S_{1} \bigcap\left(\mathbf{R}^{+} \backslash N_{\varphi}(W, \delta)\right), V \bigcap \varphi_{t}(V) \neq \emptyset$ and $\operatorname{diam}\left(\varphi_{t}(V)\right) \leq \delta$, and so

$$
V \bigcup \varphi_{t}(V) \subset \bar{B}(x, 2 \delta) .
$$

Since $\varphi$ is continuous and $\bar{B}(x, 2 \delta)$ is compact, $\left.\varphi\right|_{[0,1] \times \bar{B}(x, 2 \delta)}$ is uniformly continuous. Hence, there exists a constant $0<\varepsilon^{\prime}<$ $\min \{\varepsilon, 1\}$ such that

$$
\varphi_{t}(\bar{B}(x, 2 \delta)) \subset B(x, 3 \delta), \quad \forall t \in\left[0, \varepsilon^{\prime}\right],
$$

which, together with (40), implies that

$$
\varphi_{\tau}(V) \subset B(x, 3 \delta)
$$

for any $t \in S_{1} \cap\left(\mathbf{R}^{+} \backslash N_{\varphi}(W, \delta)\right)$ and any $\tau \in\left[t, t+\varepsilon^{\prime}\right]$. Let $U=X \backslash \bar{B}(x, 3 \delta)$ and

$$
S_{2}=\bigcup_{t \in S_{1} \cap\left(\mathbf{R}^{+} \backslash N_{\varphi}(W, \delta)\right)}\left[t, t+\varepsilon^{\prime}\right] .
$$


It follows from (42) that, for any $\tau \in S_{2}, U \cap \varphi_{\tau}(V)=\emptyset$; that is, $V \cap \varphi_{\tau}^{-1}(U)=\emptyset$. Thus,

$$
S_{1} \bigcap\left(\mathbf{R}^{+} \backslash N_{\varphi}(W, \delta)\right) \subset S_{2} \subset S .
$$

As the lower density of $S_{1}$ is positive which implies that the upper density of

$$
S \cap\left(\mathbf{R}^{+} \backslash N_{\varphi}(W, \delta)\right)
$$

is positive, since the upper density of

$$
\mathbf{R}^{+} \backslash N_{\varphi}(W, \delta)
$$

is 1 , therefore, the proof is complete.

Theorem 30. Let $(X, d)$ be a nontrivial metric space, whose bounded and closed subsets are compact, and let $\varphi$ be a continuous measure-preserving semiflow on $(X, d, \mathscr{B}(X), \mu)$. If $\varphi$ is weak mixing and $\operatorname{supp} \mu=X$, then $\varphi$ is ergodically sensitive in $X$.

Proof. On the contrary, $\varphi$ is not ergodically sensitive in $X$. Then, by Lemma 29, there exist two disjoint and nonempty open sets $U, V$ in $X$ such that the set

$$
S=\left\{t \in \mathbf{R}^{+}: V \bigcap \varphi_{t}^{-1}(U)=\emptyset\right\}
$$

has a positive upper density. Since supp $\mu=X$, we have $\mu(V) \mu(U)>0$, and so

$$
\begin{gathered}
\lim _{t \rightarrow \infty} \frac{1}{t} \int_{0}^{t}\left|\mu\left(V \bigcap \varphi_{s}^{-1}(U)\right)-\mu(V) \mu(U)\right| d s \\
\geq \mu(V) \mu(U) \bar{d}(S)>0 .
\end{gathered}
$$

This contradicts the assumption that $\varphi$ is weak mixing. Therefore, $\varphi$ is ergodically sensitive in $X$. This completes the proof.

\section{Relationships between Sensitive Properties of a Map and Its Iterations}

In the final section, we discuss relationships between sensitive properties of a map $f$ and its iterations $f^{m}$, including sensitivity, syndetic sensitivity, ergodic sensitivity, cofinite sensitivity, multisensitivity, and $n$-sensitivity. These relationships are equivalent in the special case that the space is compact and the map $f$ is continuous.

Theorem 31. Let $f: X \rightarrow X$ be a map, where $(X, d)$ is a metric space. If $f^{m}$ is sensitive or syndetically sensitive or ergodically sensitive or multisensitive in $X$ for some $m \geq 2$, then so is $f$. Moreover, the converses of all the above conclusions are true if $f$ is uniformly continuous in X. In addition, if $f$ is cofinitely sensitive, then so is $f^{m}$ for any $m \geq 2$; and if $f$ is uniformly continuous in $X$ and $f^{m}$ is cofinitely sensitive for some $m \geq 2$, then $f$ is cofinitely sensitive.

Proof. The proof is divided into four parts.
(1) It is evident that for any nonempty open set $U \subset X$ and for any constant $\gamma>0$,

$$
m N_{f^{m}}(U, \gamma) \subset N_{f}(U, \gamma)
$$

which implies that if $f^{m}$ is sensitive or syndetically sensitive or ergodically sensitive or multisensitive in $X$, then so is $f$ by the related definitions.

(2) Suppose that $f$ is uniformly continuous in $X$ and fix any integer $m \geq 2$. Then $f^{i}, 1 \leq i \leq m-1$, are uniformly continuous in $X$. Let $\delta>0$ be a constant of sensitivity with respect to one of the five types of sensitivity. Then there exists a positive constant $\varepsilon<\delta$ such that whenever $d(x, y) \leq \varepsilon$ for $x, y \in X$, one has

$$
d\left(f^{i}(x), f^{i}(y)\right) \leq \delta, \quad 0 \leq i \leq m-1 .
$$

This claim will be often used in the sequent discussion, which is divided into four steps.

Step 1. If $f$ is sensitive in $X$, then so is $f^{m}$.

Let $U \subset X$ be any nonempty open set. Since $f$ is sensitive in $X, N_{f}(U, \delta)$ is not empty. Fix any $n \in N_{f}(U, \delta)$ and then there exist $x, y \in U$ such that $d\left(f^{n}(x), f^{n}(y)\right)>\delta$. Let $n=k m+r$ with $0 \leq r \leq m-1$ and $k \in \mathbf{Z}^{+}$. Then $d\left(f^{k m}(x), f^{k m}(y)\right)>\varepsilon$. Otherwise, if $d\left(f^{k m}(x), f^{k m}(y)\right) \leq \varepsilon$, then it follows from (14) that $d\left(f^{n}(x), f^{n}(y)\right) \leq \delta$, which is a contradiction. Hence, $k \in N_{f^{m}}(U, \varepsilon)$ and so $N_{f^{m}}(U, \varepsilon) \neq \emptyset$. Therefore, $f^{m}$ is sensitive in $X$.

Step 2. If $f$ is syndetically sensitive in $X$, then so is $f^{m}$.

Let $U \subset X$ be any nonempty open set. Since $f$ is syndetically sensitive in $X, N_{f}(U, \delta)$ is syndetic in $\mathbf{Z}^{+}$. Write $N_{f}(U, \delta)$ as an increasing sequence $\left\{n_{i}\right\}_{i=1}^{\infty}$. There exists an integer $M \geq 1$ such that $n_{i+1}-n_{i}<M$ for all $i \geq 1$. Let

$$
n_{i}=m k_{i}+r_{i}, \quad i \geq 1
$$

with $0 \leq r_{i} \leq m-1$ and $k_{i} \in \mathbf{Z}^{+}$. Then

$$
n_{i+1}-n_{i}=m\left(k_{i+1}-k_{i}\right)+r_{i+1}-r_{i}<M \text {, }
$$

which implies that

$$
m\left(k_{i+1}-k_{i}\right)<M+r_{i}-r_{i+1}<M+m, \quad i \geq 1 .
$$

Thus, $\left\{k_{i}\right\}_{i=1}^{\infty}$ is syndetic in $\mathbf{Z}^{+}$. In addition, As is shown in Step $1, k_{i} \in N_{f^{m}}(U, \varepsilon)$ by the definition of $\varepsilon$. Hence, $N_{f^{m}}(U, \varepsilon)$ is syndetic, and consequently $f^{m}$ is syndetically sensitive in $X$.

Step 3. If $f$ is ergodically sensitive in $X$, then so is $f^{m}$.

Let $U \subset X$ be any nonempty open set. Then $\bar{d}\left(\mathrm{~N}_{f}(U, \delta)\right)>$ 0 . For any given $k \in N_{f}(U, \delta)$, let $k=m i_{k}+r_{k}$ with $0 \leq r_{k} \leq$ 
$m-1$ and $i_{k} \in \mathbf{Z}^{+}$. Then $i_{k} \in N_{f^{m}}(U, \varepsilon)$ as is shown in Step 1 . Consequently, we have

$$
\begin{aligned}
& m \underset{k \rightarrow \infty}{\lim \sup _{k}} \frac{\left|N_{f^{m}}(U, \varepsilon) \bigcap \mathbf{N}_{i_{k}}\right|}{i_{k}} \\
& \quad \geq \lim _{N_{f}(U, \delta) \ni k \rightarrow \infty} \frac{\left|N_{f}(U, \delta) \bigcap \mathbf{N}_{k+1}\right|}{k+1}=\bar{d}\left(N_{f}(U, \delta)\right)>0,
\end{aligned}
$$

which implies that

$$
\bar{d}\left(N_{f^{m}}(U, \varepsilon)\right) \geq \limsup _{k \rightarrow \infty} \frac{\left|N_{f^{m}}(U, \varepsilon) \bigcap \mathbf{N}_{i_{k}}\right|}{i_{k}}>0 .
$$

Therefore, $f^{m}$ is ergodically sensitive in $X$.

Step 4. If $f$ is multisensitive in $X$, then so is $f^{m}$.

Since $f$ is multisensitive in $X$, for each integer $k \geq 1$ and any $k$ nonempty open sets $V_{i} \subset X, 1 \leq i \leq k$, we have

$$
\bigcap_{i=1}^{k} N_{f}\left(V_{i}, \delta\right) \neq \emptyset
$$

Let $n \in \bigcap_{i=1}^{k} N_{f}\left(V_{i}, \delta\right)$ and $n=l m+r$ with $0 \leq r \leq m-1$ and $l \in \mathbf{Z}^{+}$. It can be easily shown that $l \in \bigcap_{i=1}^{k} N_{f^{m}}\left(V_{i}, \varepsilon\right)$ by (14), and consequently

$$
\bigcap_{i=1}^{k} N_{f^{m}}\left(V_{i}, \varepsilon\right) \neq \emptyset .
$$

Therefore, $f^{m}$ is multisensitive in $X$.

(3) If $f$ is cofinitely sensitive in $X$, then so is $f^{m}$ for any $m \geq 2$.

Let $\delta>0$ be a constant of cofinite sensitivity, $U \subset X$ any nonempty open set, and $m \geq 2$ any integer. Then there exists an integer $M \geq 1$ such that

$$
N_{f}(U, \delta) \supset[M,+\infty) \bigcap \mathbf{N} .
$$

For any integer $n \geq M$, it is clear that $n m \in N_{f}(U, \delta)$ and so there exist two points $x, y \in U$ such that

$$
d\left(f^{n m}(x), f^{n m}(y)\right)>\delta
$$

which yields that $n \in N_{f^{m}}(U, \delta)$, and consequently we get that

$$
N_{f^{m}}(U, \delta) \supset[M,+\infty) \bigcap \mathbf{N}
$$

Hence, $f^{m}$ is cofinitely sensitive in $X$.

(4) If $f$ is uniformly continuous in $X$ and $f^{m}$ is cofinitely sensitive for some $m \geq 2$, then $f$ is cofinitely sensitive.

It is clear that $f^{i}$ is uniformly continuous for $1 \leq i \leq m$. For any constant $\gamma>0$, there exists $0<\gamma^{\prime} \leq \gamma$ such that $d(x, y) \leq \gamma^{\prime}$ implies that $d\left(f^{i}(x), f^{i}(y)\right) \leq \gamma$ for $1 \leq i \leq m$.
This implies that if $n \in N_{f^{m}}(U, \gamma)$, then $m(n-1), m(n-1)+$ $1, \ldots, m n \in N_{f}\left(U, \gamma^{\prime}\right)$. This means that $\{m(n-1)+i: n \in$ $\left.N_{f^{m}}(U, \gamma), 0 \leq i \leq m\right\} \subset N_{f}\left(U, \gamma^{\prime}\right)$. Hence, $f$ is cofinitely sensitive.

The entire proof is complete.

Theorem 32. Let $f: X \rightarrow X$ be a map, where $(X, d)$ is a nontrivial metric space. For any given $m \geq 2$, if $f^{m}$ is $n$ sensitive in $X$, then so is $f$. Moreover, the converses of the above conclusion are true if $f$ is uniformly continuous in $X$.

Proof. The proof is similar to that of Theorem 31 and is omitted.

Remark 33. In the study of topological dynamical systems, it is the most important case that the space $X$ is compact and the map $f$ continuously transforms the space into itself. Clearly, $f$ is uniformly continuous in $X$, and consequently $f$ is sensitive, syndetically sensitive, ergodically sensitive, cofinitely sensitive, $n$-sensitive, and multisensitive in $X$ if and only if so is $f^{m}$, respectively, for any given integer $m \geq 2$ in this case.

\section{Conflict of Interests}

The authors have declared that there is no conflict of interests regarding the publication of this paper.

\section{Acknowledgments}

The authors sincerely thank the referees for their careful reading and useful remarks, which helped us improve the paper. This research was supported by the NNSF of Shandong Province (Grant ZR2011AM002).

\section{References}

[1] C. Abraham, G. Biau, and B. Cadre, "Chaotic properties of mappings on a probability space," Journal of Mathematical Analysis and Applications, vol. 266, no. 2, pp. 420-431, 2002.

[2] C. Abraham, G. Biau, and B. Cadre, "On Lyapunov exponent and sensitivity for maps of the interval," Tech. Rep., University Montpellier II, 2001.

[3] J. Banks, J. Brooks, G. Cairns, G. Davis, and P. Stacey, "On Devaney's definition of chaos," The American Mathematical Monthly, vol. 99, no. 4, pp. 332-334, 1992.

[4] B. Cadre and P. Jacob, "On pairwise sensitivity," Journal of Mathematical Analysis and Applications, vol. 309, no. 1, pp. 375382, 2005.

[5] R. L. Devaney, An Introduction to Chaotic Dynamical Systems, Addison-Wesley, New York, NY, USA, 2nd edition, 1989.

[6] E. Glasner and B. Weiss, "Sensitive dependence on initial conditions," Nonlinearity, vol. 6, no. 6, pp. 1067-1075, 1993.

[7] B. Hasselblatt and A. Katok, A First Course in Dynamics, Cambridge University Press, Cambridge, UK, 2003.

[8] L. He, X. Yan, and L. Wang, "Weak-mixing implies sensitive dependence," Journal of Mathematical Analysis and Applications, vol. 299, no. 1, pp. 300-304, 2004. 
[9] W. Huang, P. Lu, and X. Ye, "Measure-theoretical sensitivity and equicontinuity," Israel Journal of Mathematics, vol. 183, pp. 233283, 2011.

[10] J. James, T. Koberda, K. Lindsey, C. E. Silva, and P. Speh, "Measurable sensitivity," Proceedings of the American Mathematical Society, vol. 136, no. 10, pp. 3549-3559, 2008.

[11] R. Li and Y. Shi, "Several sufficient conditions for sensitive dependence on initial conditions," Nonlinear Analysis. Theory, Methods \& Applications. An International Multidisciplinary Journal. Series A: Theory and Methods, vol. 72, no. 5, pp. 27162720, 2010.

[12] T. K. S. Moothathu, "Stronger forms of sensitivity for dynamical systems," Nonlinearity, vol. 20, no. 9, pp. 2115-2126, 2007.

[13] S. Shao, X. Ye, and R. Zhang, "Sensitivity and regionally proximal relation in minimal systems," Science in China. Series A. Mathematics, vol. 51, no. 6, pp. 987-994, 2008.

[14] J. Xiong, "Chaos in a topologically transitive system," Science in China. Series A. Mathematics, vol. 48, no. 7, pp. 929-939, 2005.

[15] V. V. Nemiskii and V. V. Stepanov, Qualitative Theory of Ordinary Differential Equations, Princeton University Press, Princeton, NJ, USA, 1960.

[16] X. Ye and R. Zhang, "On sensitive sets in topological dynamics," Nonlinearity, vol. 21, no. 7, pp. 1601-1620, 2008.

[17] E. Akin and S. Kolyada, "Li-Yorke sensitivity," Nonlinearity, vol. 16, no. 4, pp. 1421-1433, 2003.

[18] J. Li, S. Tu, and X. Ye, "Mean equicontinuity and mean sensitivity," Ergodic Theory and Dynamical Systems, http://arxiv.org/abs/1312.7663 .

[19] K. Petersen, Ergodic Theory, vol. 2, Cambridge University Press, Cambridge, UK, 1983.

[20] P. Walters, An Introduction to Ergodic Theory, vol. 79, Springer, New York, NY, USA, 1982.

[21] E. Akin, The General Topology of Dynamical Systems, vol. 1 of Graduate Studies in Mathematics, The American Mathematical Society, Providence, RI, USA, 1993.

[22] S. Lardjane, "On some stochastic properties in Devaney's chaos," Chaos, Solitons and Fractals, vol. 28, no. 3, pp. 668-672, 2006. 


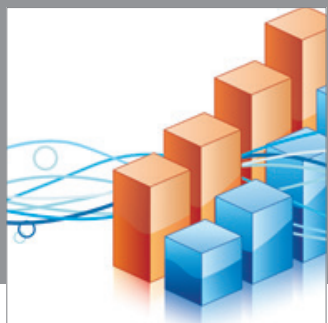

Advances in

Operations Research

mansans

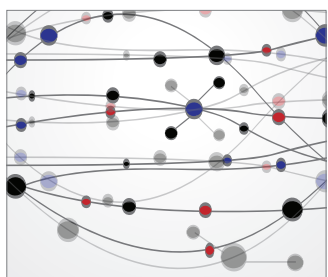

The Scientific World Journal
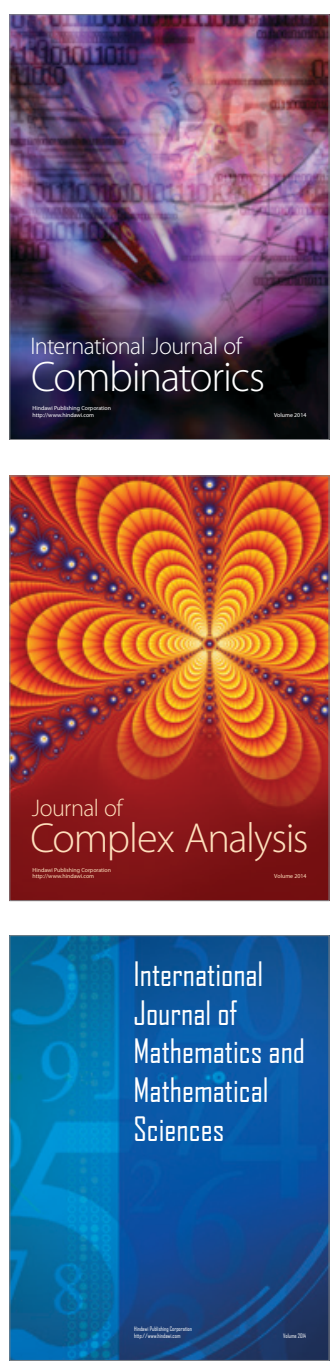
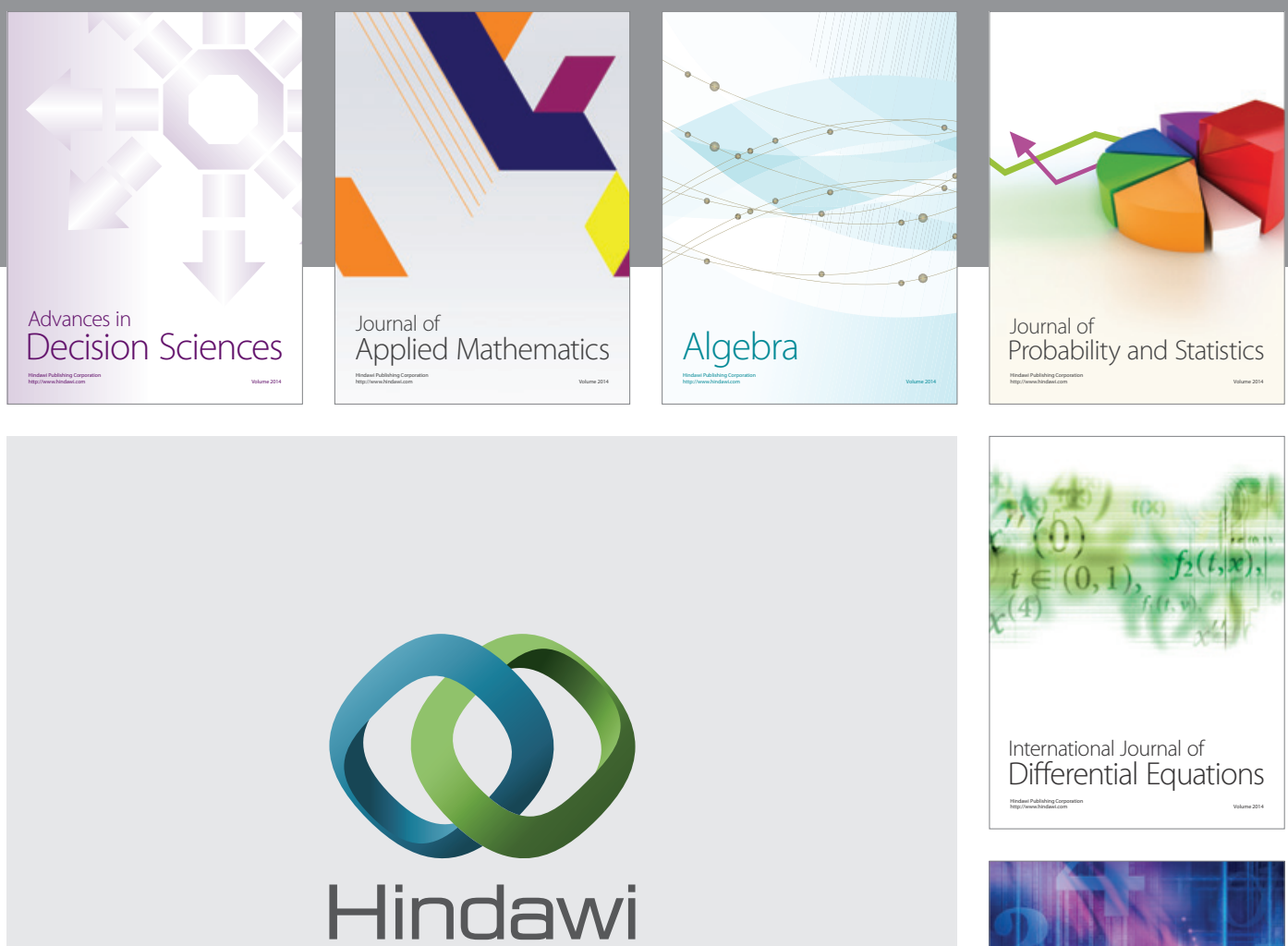

Submit your manuscripts at http://www.hindawi.com
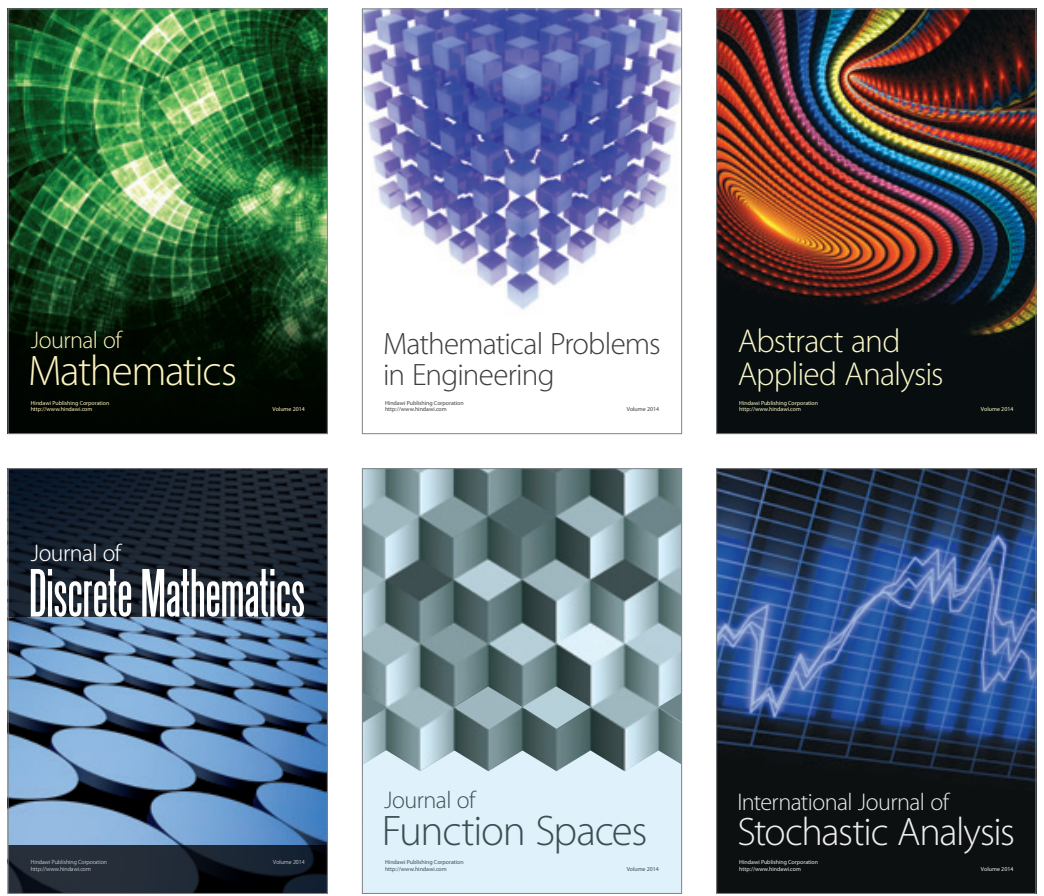

Journal of

Function Spaces

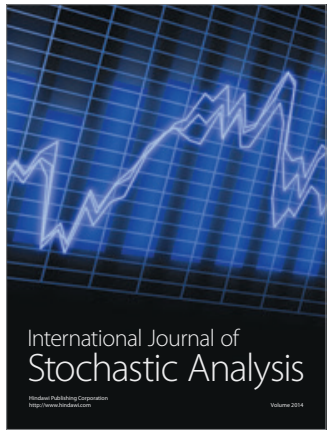

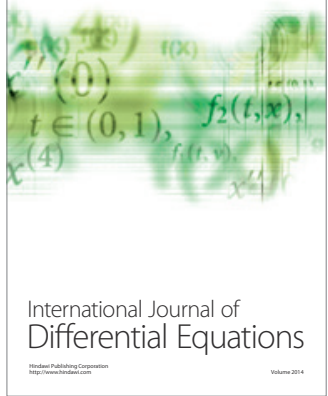
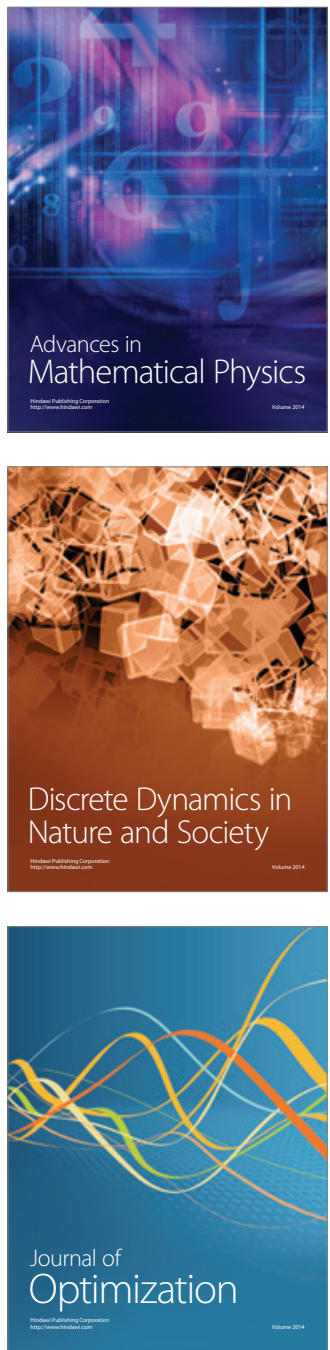